Disponível em

http://www.anpad.org.br/rac

RAC, Rio de Janeiro, v. 19, n. 6, art. 3, pp. 712-731, Nov./Dez. 2015

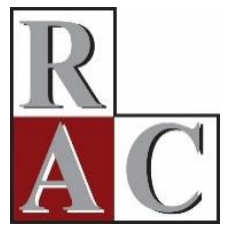

\title{
Orgulho de Ser Brasileiro Impacta o Nível de Felicidade?
}

Does Pride of Being Brazilian Impact Happiness?

Luciana Massaro Onusic Universidade Federal de São Paulo - UNIFESP/EPPEN

Wesley Mendes-da-Silva Fundação Getulio Vargas - FGV/ Eaesp 


\title{
Resumo
}

Na última década tem-se verificado um expressivo crescimento do número de trabalhos dedicados à identificação de determinantes do bem-estar subjetivo dos indivíduos. Contudo, um importante preditor do bem-estar tem sido pouco estudado, o orgulho nacional, em especial nos países latino-americanos. A partir de uma abordagem comparada entre o Brasil e demais países latino-americanos, este artigo verifica a existência de associações entre o sentimento de orgulho nacional e o nível de bem-estar dos indivíduos. Para tanto, foram empregados modelos de resposta binária e dados pertencentes a um survey realizado anualmente pelo Latinobarómetro, relativos aos anos 2000 e 2009. Os principais resultados sugerem que no Brasil, em 2009, quanto maior o orgulho de ser brasileiro, maior a probabilidade do indivíduo se declarar muito feliz, o que não ocorria em 2000, enquanto nos demais países latino-americanos isso já era contemplado. Esses resultados sugerem o aumento da relevância do orgulho nacional para o nível de bem-estar da população brasileira.

Palavras-chave: orgulho nacional; felicidade; bem-estar; satisfação com a vida; América Latina.

\begin{abstract}
Using a comparative approach between Brazil and other Latin American countries, this article aims to analyze the association of the national pride with individuals' well-being. For this end, we used the Latinobarómetro database for the years 2000 and 2009. The Latinobarómetro is a public opinion survey conducted by a non-governmental organization based in Santiago, Chile, that applies a questionnaire in 18 Latin American countries with about 20,000 respondents representing about 600 million residents. From simulations using binary response (logit) models, the main results suggest that in 2009 in Brazil, pride in being Brazilian started to have an effect on the probability a person reports being very happy, which did not occur in 2000 . This was different from other Latin American countries in 2000, where people did report being very happy.
\end{abstract}

Key words: national pride; happiness; well being; life satisfaction; Latin America. 


\section{Introdução}

Nos últimos anos, o tema da felicidade tem despertado a atenção dos governos ao redor do mundo. Em alguns países como Japão, Coreia do Sul, Inglaterra e França, já é um dos componentes dos indicadores de desempenho de gestão de políticas públicas, além de constituir tema relevante no estudo dos determinantes da produtividade das pessoas (Mendes-da-Silva, Onusic, Norvilitis, \& Moura, 2013; Oswald, Proto, \& Sgroi, 2014). Um reflexo disso é o crescente interesse de diversos organismos internacionais no melhor entendimento dos determinantes da felicidade ao redor do mundo (Helliwell, Layard, \& Sachs, 2013; OECD Better Life Index, 2014). Nesse sentido, Nicolas Sarkozy, então Presidente da França, realçou os argumentos de Joseph Stiglitz, para quem o êxito de uma economia não deve ser medido apenas pelo Produto Interno Bruto (PIB), mas também deve refletir aspectos de bemestar (felicidade) dos indivíduos.

Essa mesma linha de pensamento vem sendo compartilhada por pesquisadores e formadores de políticas públicas do Reino Unido (London Datastore, n.d.; Oswald, 1997). No caso brasileiro, já é possível encontrar iniciativas nessa direção. Um exemplo é a Proposta de Emenda à Constituição no 19 de 2010, que ficou conhecida por PEC da Felicidade e que propõe alterações no art. $6^{\circ}$ da Constituição Federal para considerar os direitos ali previstos como direitos sociais essenciais à busca do bem-estar. Contudo, a esse respeito, Diener e Chan (2011) destacam que políticas públicas ainda são predominantemente definidas com base em métricas objetivas, tais como expectativa de vida e índices de mortalidade infantil.

Uma expressiva parcela da literatura tem dedicado atenção à identificação dos determinantes do bem-estar subjetivo dos indivíduos, ou seja, o julgamento de satisfação geral com a vida, ou felicidade (Dolan, Peasgood, \& White, 2008; Kahneman, 1999; Lykken \& Tellegen, 1996; Scorsolini-Comin \& Santos, 2010), incluindo-se trabalhos recentemente realizados no Brasil (Corbi \& Menezes-Filho, 2006; Mendes-da-Silva et al., 2013). Entre os determinantes da felicidade, um dos que têm recebido crescente atenção é o orgulho nacional, tal como discutem Ha e Jang (2015). Contudo, a associação entre felicidade e a noção de orgulho nacional apresenta-se, ao mesmo tempo, relevante e pouco estudada. A esse respeito, estudos recentes, como o de Morrison, Tay e Diener (2011), partem do argumento central da Teoria da Identidade Social (Tajfel, 1981). Por sua vez, segundo essa linha de pensamento, um importante componente da identidade, e da autoestima dos indivíduos, é o fato de participar e pertencer a um grupo.

Para Corbi e Menezes-Filho (2006), as pesquisas sobre bem-estar, ou felicidade, são caracterizadas por uma abordagem conexa, segundo a qual são empregados repertórios teóricoconceituais pertencentes a diferentes campos de conhecimento, tais como: Psicologia, Medicina, Economia, Geografia e Finanças. A esse respeito, Diener e Seligman (2004) assumem que um componente representativo de uma boa vida é a felicidade. Segundo tais autores, a natureza da felicidade não é uniformemente definida. Felicidade pode sugerir prazer, satisfação de vida, emoções positivas, sensação de contentamento, entre outros conceitos. Em síntese, felicidade não é um conceito claramente distinto de prazer, satisfação ou bem-estar. Na presente pesquisa, tal como adotado por Schkade e Kahneman (1998), e recentemente no Brasil, por Mendes-da-Silva, Onusic, Norvilitis e Moura (2013), assume-se felicidade como sinônimo de satisfação com a vida e de bem-estar. Na busca da identificação de determinantes do bem-estar, têm-se apontado aspectos diversos, entre eles, um dos mais recentes é o orgulho de seu país de origem (Reeskens \& Wright, 2011). Na última década, a literatura tem documentado pesquisas a partir de economias desenvolvidas e em desenvolvimento (Evans \& Kelley, 2002). A esse respeito, a literatura documenta evidências da África do Sul, segundo a qual o orgulho nacional e a felicidade se relacionam devido à sensação de unidade nacional e de confiança no futuro (Moller, Dickow, \& Harris, 1999); bem como recente estudo realizado na Coreia do Sul (Ha \& Jang, 2015), que apresenta evidências que sugerem que o orgulho nacional está associado positivamente à felicidade. 
Com base nesses argumentos, na relevância do tema da felicidade e, ainda, na escassez de estudos ao redor dessa temática, o objetivo central deste trabalho é verificar a existência de associações entre o orgulho nacional e o bem-estar dos indíviduos no Brasil, a partir de uma abordagem comparada aos demais países pertencentes à América Latina. Para tanto, foram empregados modelos de resposta binária, com base em dados coletados e disponibilizados pelo Latinobarómetro, relativos aos anos 2000 e 2009. Este artigo está estruturado em sete seções, sendo esta Introdução a primeira delas. A segunda seção discute o contexto econômico corrente na América Latina e no Brasil, por ocasião da coleta dos dados empregados nesta pesquisa. Em seguida, a terceira seção apresenta a revisão de literatura acerca dos determinantes da felicidade. Já a quarta seção deste artigo concentra-se na discussão do papel do orgulho nacional como determinante da felicidade. Os procedimentos metodológicos adotados nesta pesquisa são detalhados na quinta seção. A sexta seção apresenta a discussão dos resultados empíricos alcançados. E, finalmente, as considerações finais são apresentadas na sétima e última seção.

\section{Contexto na América Latina e no Brasil}

Para contextualizar a atualidade política/econômica da América Latina, vale retomar o histórico abordado no trabalho de Cano (2009) que relata que desde o fim da II Guerra Mundial, a priorização conferida pelos Estados Unidos da América (EUA) foi em relação à reconstrução da Europa, tendo como consequência menor relevância destinada aos esforços dirigidos à América Latina, em termos de suas decisões políticas. No entanto, até a década de 1970, observou-se uma gama de alterações do ambiente político em vários países latino-americanos, predominando regimes caracterizados por intervenções militares e relativa instabilidade econômica entre esses países, nesse período. Segundo Cano (2009):

Entre 1980 e 2003 continuamos estagnados: até o início da década de 1990 com alta inflação, e, a partir daí, com os ajustes neoliberais, com baixa inflação, mas com alto desemprego. De 2003 a 2008, vivemos a ilusão da falsa recuperação do processo de desenvolvimento, graças ao chamado "efeito China". Mas a ilusão se desfez já desde meados de 2008, com a desaceleração do comércio internacional e com a "crise anunciada" do mercado financeiro nos EUA. Tivemos, ao longo de todo esse período, não só baixo crescimento médio do PIB e do emprego, mas também sua precarização e fortes indícios de desindustrialização (Unctad, 2003). Daí considerarmos o curto período 2003-2008 como excepcional, pois estamos convivendo com uma crise estrutural de quase 30 anos! (p. 606).

No Brasil, desde 1999, foi instituído o Sistema de Metas de Inflação no Brasil, estabelecendo que a Autoridade Monetária deveria perseguir um único objetivo, o controle da inflação. No entanto, o sistema teve dificuldade de mantê-la no início, mas a partir de 2004 manteve o controle com sucesso, até mesmo na crise de 2008 .

Segundo Baer (2014) e Serrano e Summa (2011), a taxa de crescimento do Brasil entre 1999 e 2003 mostrou-se relativamente reduzida. A partir de 2006, as taxas de exportação caem e o crescimento interno do país ganha destaque, devido a uma política fiscal mais expansionista adotada pelo governo. Assim, tem-se que, após 2005, a renda familiar média começa a crescer devido a salários reais médios mais altos, bem como o crescimento mais rápido da economia e dos empregos formais. A parcela dos pobres e extremamente pobres era estável até 2003. A partir de 2004, e mesmo na crise (recessão) de 2009, estas parcelas foram sendo reduzidas. Com a crise de 2008, a taxa de crescimento cai, porém recupera-se no final de 2009. Entre 2004 e 2010, as metas de inflação foram alcançadas, com algum crescimento da economia brasileira. 


\section{Determinantes da Felicidade}

Para os economistas, a felicidade geralmente não está definida, mas pode ser empiricamente medida com base em respostas coletadas com uso de questionários, nos quais as pessoas respondem "o quão feliz você está?" Um exemplo dessa visão pode ser encontrado no trabalho de Inglehart (1996, p. 516). Entre as variáveis mais frequentemente estudadas como determinante da felicidade (ou bem-estar, ou satisfação com a vida) está a renda dos indivíduos (Boyce, Brown, \& Moore, 2010). Pesquisadores como Easterlin (2001), Oswald (1997) e Frey e Stutzer (2002) têm documentado associação positiva entre renda e felicidade.

Mas, a respeito da natureza e das causas da felicidade, dois psicólogos, Brickman e Campbell (1971), ressaltam um paradoxo entre riqueza (financeira) e felicidade. Segundo esses autores, existiriam níveis além dos quais o incremento de renda não ocasionaria aumento de satisfação com a vida. Nesse sentido, Corbi e Menezes-Filho (2006) argumentam que existe a possibilidade que níveis mais elevados de renda não trariam felicidade para as pessoas, e também que as pessoas mais felizes ganhem mais dinheiro porque elas tendem a trabalhar mais arduamente, além de serem mais empreendedoras. Em adição, na visão de Lykken e Tellegen (1996) e de Kahneman (1999), uma questão que parece não ser contestável à luz dos trabalhos acerca da felicidade, seja no contexto da pesquisa em Ciências Sociais, Economia, ou Psicologia, é que se trata de uma questão de julgamento das pessoas, portanto, trata-se de uma questão subjetiva.

No Brasil, ainda pouco se sabe a respeito de evidências empíricas dos determinantes de felicidade do indivíduo. Uma pesquisa realizada por Mendes-da-Silva et al. (2013) analisa as associações entre a satisfação com aspectos de vida nas cidades e a satisfação geral com a vida, enfatizando a ocorrência de viés de julgamento na percepção de satisfação com a vida. Os resultados da pesquisa sugerem que as pessoas tendem a superestimar a influência de determinados aspectos particulares de vida (tal como segurança pessoal) sobre seu bem-estar. Nessa mesma linha de argumento, resultados obtidos por Oswald (1997) sugerem que a insatisfação com a vida pode aumentar significativamente a ocorrência de suicídios. Porém, convém destacar que, segundo esse autor, nas regiões nas quais se verificam melhores condições de vida, como por exemplo nos países nórdicos, a ocorrência de suicídios seria mais sensível a níveis menores de satisfação com a vida, em que pese se tratar de países de elevado padrão de desenvolvimento. Ainda a esse respeito, nas regiões de menor taxa de desemprego, por exemplo, haveria maior probabilidade de um indivíduo desempregado cometer suicídio (Platt, Micciolo, \& Tansella, 1992). No contexto brasileiro, Mendes-da-Silva et al. (2013) ressaltam que o Rio Grande do Sul, ao mesmo tempo em que constitui uma das regiões de maior nível de desenvolvimento, evidencia uma das maiores taxas de morte por suicídio no país.

Pesquisadores como Edgerton (1992), Graham (2008), Veenhoven (2008) e Diener e Chan (2011) discutem as associações entre felicidade, saúde e longevidade. Desse modo, entende-se que tratar do tema felicidade da população pode contribuir para o melhor entendimento de questões relevantes para o delineamento de políticas de saúde pública (Luz, César, Lima-Costa, \& Proietti, 2011). Jeon, Black, Govett, Yen e McRae (2012), a partir de dados oriundos da Austrália, concluem que as dificuldades financeiras podem afetar a qualidade de vida dos idosos, uma vez que, em função da necessidade de arcar com despesas pessoais, como pagamento de seguro saúde, relegam ao segundo plano atividades essenciais ao seu bem-estar, tal como o envolvimento em atividades sociais.

A satisfação com a vida apresenta-se sensível ao perfil dos indivíduos. Assim, desde a própria definição do que pode ser visto como felicidade, até variáveis sociodemográficas, podem influenciar a satisfação com a vida. Em se tratando do público jovem, a literatura sugere que a satisfação com a vida está significativamente associada a perspectivas de trabalho, oportunidades de crescimento intelectual, situação financeira e segurança pessoal (Lichtenstein \& Slovic, 2006; Loewenstein, Read, \& Baumeister, 2003). Já as pessoas mais idosas, segundo Burr, Santo e Pushkar (2011), tendem a dispor de mais tempo para dedicar-se aos amigos e à família, não sofrem a pressão do mercado de trabalho, além de que, frequentemente, não se percebem na responsabilidade de planejar o futuro. 
Nesse contexto, encontram-se pesquisas acerca de temas como sociabilidade dos indivíduos, comportamento de consumo e poupança, relação das pessoas com o dinheiro, afetividade e orgulho do país (Ahuvia \& Friedman, 1998; Diener \& Seligman, 2004; Duesenberry, 1949; Kahneman, Krueger, Schkade, Schwarz, \& Stone, 2006; Ng, 1997; Schildkraut, 2014).

\section{Orgulho Nacional Como Determinante da Felicidade}

O sentimento de identidade nacional, bem como suas causas e suas consequências, tem sido alvo de estudo. Em outras palavras, o grau de apego a ser de determinado país tem sido apontado como algo relevante, seja exercendo o papel de variável dependente, como variável independente (Denham, 2010; Kavetsos, 2012; Schildkraut, 2014). No presente estudo, adota-se o sentimento de identidade nacional como determinante da felicidade (ou bem-estar, ou satisfação com a vida), o que não é necessariamente uma novidade na literatura (Moller et al., 1999). No entanto, acerca do contexto latino-americano, especialmente a respeito do Brasil, poucos estudos têm analisado o problema sob exame no presente trabalho.

Para Morrison et al. (2011), o sentimento de orgulho do país, ou nacionalismo, é um dos fatores relevantes que contribuem para o aumento da satisfação com a vida. Nesse estudo, a partir de um survey internacional, Morrison et al. (2011) encontraram indícios de que o orgulho nacional pode ser um forte preditor da satisfação com a vida, e que esta última guarda relação moderada com: renda familiar, mobilidade residencial, Produto Interno Bruto per-capta e região (país não-ocidental e ocidental). Em adição, nesse mesmo estudo, foram encontrados resultados que apontam que, entre os indivíduos mais empobrecidos, o orgulho nacional está mais fortemente associado à satisfação com a vida.

Dito de outra forma: indivíduos em situação de acentuada pobreza podem elevar o pertencimento à nação a um componente mais central de sua identidade social, tornando-o mais relevante ao julgar sua qualidade de vida. Morrison et al. (2011) destacam ainda que, satisfação com a vida e orgulho nacional estão mais fortemente associados quando considerados os países não-ocidentais. Além disso, os resultados sugerem que a satisfação com saúde, padrão de vida e trabalho parecem ser mais importantes para a satisfação com a vida entre os indívíduos mais ricos.

No entanto, Reeskens e Wright (2011) questionam se o sentimento de orgulho nacional contribui para o nível de felicidade do indivíduo e se propõem a elencar tipos diferentes de orgulho nacional. Reeskens e Wright (2011) classificam o orgulho nacional em duas classes principais: (a) nacionalismo étnico - expresso geralmente em termos religiosos ou raciais (segundo Pehrson et al., 2009, este sentimento pode incentivar a xenofobia, o sentimento autoritário, e isto pode reduzir o sentimento de bem-estar) e (b) nacionalismo cívico - este é mais abrangente, representado pelo respeito às instituições do país e às leis vigentes, estando mais aberto a minorias, incluindo-se imigrantes. Ou seja, a literatura aponta, ainda, que o nacionalismo cívico contém elementos de universalismo e benevolência. E, por isso, dele espera-se incremento dos níveis de felicidade dos indivíduos (Deneve \& Cooper, 1998; Sagiv \& Schwartz, 2000; Schwartz, Sagiv, \& Boehnke, 2000).

O trabalho de Reeskens e Wright (2011) considerou dados oriundos de um survey realizado com 40.677 pessoas, em 31 países, no contexto do European Values Study, edição 2008. Esse trabalho sugere que há associação entre orgulho nacional e bem-estar pessoal. No entanto, segundo os resultados de Reeskens e Wright (2011), quem possui orgulho nacional classificado como nacionalismo cívico alcança níveis mais elevados de felicidade, se comparados aos respondentes que apresentaram orgulho nacional classificado como nacionalismo étnico.

Moller, Dickow and Harris (1999), ao analisar as associações entre orgulho nacional e felicidade, com dados coletados na África do Sul, entre 1995 e 1996, defendem que desde a primeira eleição livre, em abril de 1994, os sul africanos ficaram conhecidos como rainbow people. Segundo esse estudo, o orgulho nacional após o apartheid, a experiência com a democracia e a inclusão do tema rainbow people 
como símbolo político fizeram o povo sentir uma melhora no bem-estar subjetivo. Os resultados mostraram que sul africanos com melhores condições de vida tendem a ser mais felizes e satisfeitos com a vida, mas menos orgulhosos. De forma alternativa, os mais pobres eram menos felizes, mas muito mais orgulhosos de seu país. O estudo sugere que a crença na unidade nacional pode ser um componente importante do bem-estar pessoal e que inspira confiança no futuro, no momento de transição para uma democracia estável.

Hallmann, Breuer e Kühnreich (2013), com emprego de dados oriundos da Alemanha, analisaram quais fatores influenciam o orgulho nacional e felicidade quando atletas de sucesso participam de competições internacionais. Os resultados mostraram que 66,2\% dos entrevistados se sentiram orgulhosos e 65,6\% se autoclassificaram como felizes quando os atletas alemães alcançaram êxito em competições de elevado prestígio. Segundo os resultados obtidos por esses autores, o orgulho nacional e a felicidade podem ser explicados por meio de participação em esportes de elite e variáveis sócio econômicas.

Além disso, mulheres, indivíduos de baixa renda, assim como sujeitos de menor nível de escolaridade, tendem a ganhar mais em termos de orgulho e felicidade, em função do sucesso dos atletas. Segundo esses autores, o estudo mostra que o investimento em esportes de elite pode ser considerado um relevante instrumento de política para a integração social. De forma similar, pesquisa realizada na Coreia do Sul sugere que o orgulho nacional está associado positivamente à felicidade (Ha \& Jang, 2015).

\section{Metodologia}

\section{Coleta de dados e variáveis}

Para investigar se o orgulho nacional está associado ao nível de felicidade do indivíduo, foram empregados dados disponibilizados pelo Latinobarómetro nos anos 2000 e 2009, disponíveis no website do Latinobarómetro $(2000,2009)$, que consiste em um survey anualmente realizado em países da América Latina. Esse estudo de opinião pública considera aproximadamente 20.000 entrevistas, em 18 países da América Latina. O questionário utilizado como instrumento de coleta envolve questões relativas ao desenvolvimento da democracia, da economia e da sociedade de modo geral. Para tanto, são empregados indicadores de opinião pública que medem atitudes, valores e comportamentos. Entre essas variáveis, encontram-se indicadores relativos a: dimensões sociodemográficas, orgulho nacional, satisfação com serviços públicos, satisfação com a vida, entre outras. Mediante interesse, sugere-se examinar a íntegra do questionário, que é disponibilizado no website do Latinobarómetro, nos anos 2000 e 2009.

A Corporação Latinobarómetro é uma organização não governamental, sem fins lucrativos, com sede em Santiago do Chile. Para este estudo, os dados foram coletados em 2000 e 2009. Ao observar a Tabela 1, nota-se que o Brasil, em 2009, respondeu por aproximadamente 5,76\% dos 38.242 respondentes ao survey realizado pelo Latinobarómetro, distribuídos entre 18 países. Se observada a composição do conjunto de respondentes no ano 2000, constata-se que a quantidade de entrevistados foi sensivelmente menor, 18.038 respondentes, sendo 1.000 ( $\sim 5,54 \%$ do total de respondentes) oriundos do Brasil. As variáveis envolvidas no estudo são as seguintes:

- Sexo = Variável dummy que representa o sexo biológico do respondente. Assume valor $=1$ se masculino, e 2 no caso alternativo.

. Idade = Idade do respondente expressa em número de anos.

- Solteiro = Variável dummy que indica se o respondente é solteiro. Assume valor $=1$ se solteiro, e valor $=0$ no caso alternativo. 
- Universidade = Variável dummy que indica se o indivíduo chegou a frequentar o ensino superior, mesmo que não concluído $=1$; caso alternativo $=0$.

- Orgulho nacional $=$ Variável dummy que indica o nível de orgulho que o respondente sente de seu país, onde $1=$ Muito orgulhoso ou Razoavelmente orgulhoso; e $0=$ Pouco orgulhoso ou Nada orgulhoso.

- Renda = Variável dummy que indica se o respondente considera que sua a renda familiar é considerada suficiente para cobrir suas necessidades satisfatoriamente, onde $1=$ É suficiente, e nós conseguimos poupar ou É suficiente sem maiores problemas; e 0 = Não é suficiente e você tem problemas ou Não é suficiente e você tem grandes problemas.

Tabela 1

Países Considerados na Pesquisa

\begin{tabular}{|c|c|c|c|c|}
\hline \multirow[b]{2}{*}{ Países } & \multicolumn{2}{|c|}{2009} & \multicolumn{2}{|c|}{2000} \\
\hline & Freq. & $\%$ & Freq. & $\%$ \\
\hline Argentina & 2400 & 6,28 & 1200 & 6,653 \\
\hline Bolívia & 2280 & 5,96 & 1080 & 5,987 \\
\hline Brasil & 2204 & 5,76 & 1000 & 5,544 \\
\hline Chile & 2383 & 6,23 & 1183 & 6,558 \\
\hline Colômbia & 2400 & 6,28 & 1200 & 6,653 \\
\hline Costa Rica & 1981 & 5,18 & 981 & 5,439 \\
\hline Equador & 2400 & 6,28 & 1200 & 6,653 \\
\hline El Salvador & 2001 & 5,23 & 1001 & 5,549 \\
\hline Guatemala & 1989 & 5,20 & 989 & 5,483 \\
\hline Honduras & 1997 & 5,22 & 997 & 5,527 \\
\hline México & 2366 & 6,19 & 1166 & 6,464 \\
\hline Nicarágua & 2000 & 5,23 & 1000 & 5,544 \\
\hline Panamá & 1993 & 5,21 & 993 & 5,505 \\
\hline Paraguai & 1802 & 4,71 & 602 & 3,337 \\
\hline Peru & 2246 & 5,87 & 1046 & 5,799 \\
\hline República Dominicana & 1000 & 2,61 & n.d. & n.d. \\
\hline Uruguai & 2400 & 6,28 & 1200 & 6,653 \\
\hline Venezuela & 2400 & 6,28 & 1200 & 6,653 \\
\hline Total & 38.242 & 100 & 18.038 & 100 \\
\hline
\end{tabular}

Nota. Fonte: Cálculos dos autores com base nos dados da pesquisa.

Ao observar a Tabela 2, constata-se que o conjunto de respondentes é equilibrado entre indivíduos dos dois sexos, nota-se que a quantidade de indivíduos que apontaram estar Muito satisfeitos com vida aumentou entre 2000 (18,8\% dos respondentes) e 2009 (28,29\% dos respondentes). Já em relação ao orgulho nacional, embora seja a maioria dos respondentes, houve redução da parcela de indivíduos que se reportaram como Muito orgulhoso de seu país. Enquanto, em 2000, aproximadamente $66,6 \%$ dos respondentes alegaram encontrar-se muito orgulhosos de seu país, em 2009 esse percentual caiu para 62,67\% dos participantes do Survey do Latinobarómetro. 
Tabela 2

Frequência das Variáveis de Interesse Componentes do Modelo Empírico Adotado

\begin{tabular}{|c|c|c|c|c|}
\hline & \multicolumn{2}{|c|}{2009} & \multicolumn{2}{|c|}{2000} \\
\hline & Freq. & $\%$ & Freq. & $\%$ \\
\hline \multicolumn{5}{|l|}{ Satisfação com a vida } \\
\hline Muito satisfeito & 6420 & 28,29 & 3392 & 18,80 \\
\hline Razoavelmente satisfeito & 9907 & 43,66 & 3915 & 21,70 \\
\hline Não muito satisfeito & 5489 & 24,19 & 6430 & 35,65 \\
\hline Nada satisfeito & 750 & 3,31 & 4163 & 23,08 \\
\hline Não respondeu & 123 & 0,54 & 138 & 0,77 \\
\hline Total & 22690 & 100 & 18038 & 100 \\
\hline \multicolumn{5}{|l|}{ Sexo } \\
\hline Feminino & 11665 & 51,41 & 9271 & 51,40 \\
\hline Total & 22690 & 100 & 18038 & 100 \\
\hline \multicolumn{5}{|l|}{ Status Marital } \\
\hline Casado/vivendo com um parceiro(a) & 13251 & 58,40 & 10239 & 56,76 \\
\hline Solteiro & 6715 & 29,60 & 5690 & 31,54 \\
\hline Separado/Divorciado & 2604 & 11,48 & 1956 & 10,84 \\
\hline Não respondeu & 119 & 0,53 & 153 & 0,85 \\
\hline Total & 22690 & 100 & 18038 & 100 \\
\hline \multicolumn{5}{|l|}{ Nível intelectual } \\
\hline Sem educação formal & 2368 & 10,43 & 858 & 4,76 \\
\hline 1 a 12 anos de estudo & 16105 & 70,98 & 11389 & 63,14 \\
\hline Universitário incompleto & 1684 & 7,42 & 2161 & 11,98 \\
\hline Universitário completo & 1285 & 5,66 & 1854 & 10,28 \\
\hline Nível médio incompleto & 503 & 2,22 & 656 & 3,64 \\
\hline Nível médio completo & 729 & 3,21 & 1120 & 6,21 \\
\hline Não respondeu & 17 & 0,07 & 0 & 0 \\
\hline Total & 22690 & 100 & 18038 & 100 \\
\hline \multicolumn{5}{|l|}{ Renda subjetiva } \\
\hline É suficiente e você consegue poupar & 1764 & 7,77 & 1885 & 10,45 \\
\hline É apenas suficiente, sem maiores problemas & 8744 & 38,54 & 7249 & 40,19 \\
\hline Não é suficiente, e você encontra problemas & 8513 & 37,52 & 6337 & 35,13 \\
\hline Não é suficiente, e você tem grandes problemas & 3283 & 14,47 & 2112 & 11,71 \\
\hline Não respondeu & 241 & 1,06 & 345 & 1,91 \\
\hline Não soube responder & 146 & 0,64 & 110 & 0,61 \\
\hline Total & 22690 & 100 & 18038 & 100 \\
\hline
\end{tabular}


Tabela 2 (continuação)

\begin{tabular}{lcccc}
\hline & \multicolumn{2}{c}{$\mathbf{2 0 0 9}$} & \multicolumn{2}{c}{$\mathbf{2 0 0 0}$} \\
\cline { 2 - 5 } & Freq. & \% & Freq. & \% \\
\hline Quanto você está orgulhoso de sua nacionalidade & & & & \\
Muito orgulhoso & 14241 & 62,76 & 12007 & 66,57 \\
Razoavelmente orgulhoso & 6030 & 26,58 & 4093 & 22,69 \\
Pouco orgulhoso & 1663 & 7,33 & 1346 & 7,46 \\
Nada orgulhoso & 456 & 2,01 & 302 & 1,67 \\
Não respondeu & 57 & 0,25 & 90 & 0,50 \\
Não soube & 81 & 0,36 & 200 & 1,11 \\
Eu não tenho nacionalidade & 162 & 0,71 & 0 & 0 \\
Total & 22690 & 100 & 18038 & 100 \\
\hline
\end{tabular}

Nota. Esta tabela apresenta a frequência de resposta verificada para cada variável de interesse. A média (DP) de idade dos respondentes foi de 38,39 (15,65) anos, e de 40,75 (16,71) anos, nos anos 2000 e 2009, respectivamente. Fonte: Cálculos dos autores com base nos dados disponibilizados pelo Latinobarómetro Corporation. (2000). Latinobarómetro Survey. Recuperado de http://www.latinobarometro.org/latContents.jsp

As variáveis de controle $(V C)$ consistem na resposta apresentada na forma de escala Likert, refletindo o nível de são satisfação com: hospitais públicos, educação pública, atuação policial, sistema jurídico, áreas públicas e transporte. A escala varia entre 1 e 4 , onde $1=$ Muito satisfeito e $4=$ Nada satisfeito.

As variáveis adotadas nesta pesquisa foram selecionadas considerando-se os dados disponibilizados pelo Latinobarómetro e os argumentos presentes na literatura que afetam o objetivo deste trabalho, destacadamente os trabalhos de Moller et al. (1999); Reeskens e Wright (2011); Morrison et al. (2011); Hallmann et al. (2013) e Ha e Jang (2015).

\section{Modelos empíricos}

Neste estudo optou-se pelo emprego de modelos de resposta binária, tendo como variáveis dependentes duas proxies de felicidade dos indivíduos (Ferrer-i-Carbonell \& Frijters, 2004; Menard, 2002). As equações (1) e (2) representam os modelos logit, simulados a partir de dados disponibilizados pelo Latinobarómetro.

$$
\begin{gathered}
\pi_{i}=\varphi_{1}+\varphi_{2} \text { Sexo }_{i}+\varphi_{3} \text { Idade }_{i}+\varphi_{4} \text { Solteiro }_{i}+\varphi_{5} \text { Universidade }_{i}+\varphi_{6} \text { Renda }_{i} \\
+\varphi_{7}{\text { Orgulho } \text { nacional }_{i}+\varepsilon_{i}}
\end{gathered}
$$

A equação (1) estima a probabilidade $\pi$ de um indivíduo $i$ declarar-se Muito feliz, em função das variáveis: Sexo, Idade, Solteiro, Universidade, Renda e Orgulho nacional. O termo de erro aleatório é representado por $\varepsilon$. A equação (2) considera, além das variáveis presentes em (1), as variáveis de controle $(V C)$, representadas pela satisfação dos respondentes com aspectos particulares de vida, como: hospitais públicos, educação pública, atuação da polícia, sistema jurídico, áreas públicas e transporte.

$$
\begin{gathered}
\pi_{i}=\varphi_{1}+\varphi_{2} \text { Sexo }+\varphi_{3} \text { Idade }+\varphi_{4} \text { Solteiro }+\varphi_{5} \text { Universidade }+\varphi_{6} \text { Renda } \\
+\varphi_{7} \text { Orgulho nacional }+\sum_{j=1}^{k} \gamma_{j} V C_{j i}+\varepsilon_{i}
\end{gathered}
$$

As variáveis de controle $(V C)$, que refletem a satisfação do $i$-ésimo respondente com diferentes aspectos de vida no seu país, foram selecionadas com base na possível influência que exercem sobre a variável dependente, ou seja, sobre o nível de felicidade do i-ésimo respondente (Nível de Felicidade ${ }_{i}$ ) 
e sobre as variáveis independentes. Caso as variáveis de controle possuam correlação significante com algumas dessas variáveis, mas não sejam consideradas no modelo, a relação entre as variáveis de interesse pode não ser adequadamente evidenciada.

Assim, com base nos argumentos presentes na literatura (Ferrer-i-Carbonell \& Frijters, 2004; Ha \& Jang, 2015; Hallmann, Breuer, \& Kühnreich, 2013; Menard, 2002; Moller et al., 1999; Morrison, Tay, \& Diener, 2011; Reeskens \& Wright, 2011), bem como na disponibilidade de dados no Latinobarómetro (2000, 2009), seis variáveis de controle foram utilizadas, isto é, a satisfação com: (a) Hospitais públicos, (b) Educação pública, (c) Atuação da polícia, (d) Sistema jurídico, (e) Áreas públicas e (f) Transporte.

\section{Resultados Empíricos}

O ajustamento dos modelos estimados foi diagnosticado com base em quatro diferentes métricas: (a) $\mathrm{R}^{2}$ de MacFadden (Aldrich \& Nelson, 1984); (b) percentagem de eventos classificados corretamente; (c) Likelihood ratio; (d) Teste de homocedasticidade do termo de erro, $\varepsilon_{i}$, da regressão. As duas primeiras métricas são usadas para ilustrar o nível de ajustamento do modelo estimado. A terceira métrica indica se as variáveis independentes, conjuntamente, não possuem efeito sobre a variável dependente. E, a quarta e última métrica é um diagnóstico de verificação dos resultados obtidos.

A Tabela 3 apresenta os resultados obtidos a respeito das estimações dos coeficientes de cada variável independente componente do modelo empírico adotado para identificar a probabilidade de um indivíduo reportar-se Muito feliz. Ao explorar o conjunto de dados relativos ao Brasil e América Latina (AL), utilizados para simular o modelo nos anos de 2000 e 2009, são verificados principalmente dois resultados. Primeiro, a Renda exerce efeito persistente sobre a probabilidade de reportar-se Muito feliz, tanto no Brasil como na América Latina. Segundo, os respondentes brasileiros, entre 2000 e 2009, passaram a associar o sentimento de orgulho de ser brasileiro ao status de estar Muito feliz. Este resultado corrobora os estudos de Frey e Stutzer (2002), de Oswald (1997), bem como os de Corbi e Menezes-Filho (2006), no qual foi encontrada associação positiva entre renda e felicidade.

Tabela 3

Efeito Marginal do Perfil do Indivíduo sobre a Condição de Muito Feliz

\begin{tabular}{lllll}
\hline \multirow{2}{*}{$\begin{array}{l}\text { Variáveis independentes e fit do } \\
\text { modelo }\end{array}$} & $\begin{array}{l}\text { Coef. estimado } \\
\text { na AL }\end{array}$ & $\begin{array}{l}\text { Coef. estimado } \\
\text { no Brasil }\end{array}$ & $\begin{array}{l}\text { Coef. estimado } \\
\text { na AL }\end{array}$ & $\begin{array}{l}\text { Coef. estimado } \\
\text { no Brasil }\end{array}$ \\
\cline { 2 - 5 } Constante & $-1,670^{* * *}$ & $-1,643$ & $1,019^{* * *}$ & $-1,825$ \\
& $(0,145)$ & $(1,441)$ & $(0,211)$ & $(1,186)$ \\
Sexo $^{(\text {a) }}$ & $-0,024$ & $-1,159^{* *}$ & $-0,075$ & $-0,323$ \\
& $(0,049)$ & $(0,555)$ & $(0,085)$ & $(0,464)$ \\
Idade $^{(\text {b) }}$ & $-0,001$ & 0,025 & $-0,013^{* * *}$ & $-0,002$ \\
& $(0,002)$ & $(0,022)$ & $(0,003)$ & $(0,012)$ \\
Solteiro $^{(\text {c) }}$ & $-0,053$ & $1,243^{*}$ & 0,100 & $-0,116$ \\
& $(0,060)$ & $(0,715)$ & $(0,103)$ & $(0,553)$ \\
Frequentou Universidade $^{(\mathrm{d})}$ & $0,226^{* * *}$ & $-1,570^{*}$ & $0,744^{* * *}$ & 2,594 \\
& $(0,059)$ & $(0,819)$ & $(0,164)$ & $(1,624)$ \\
\hline
\end{tabular}


Tabela 3 (continuação)

\begin{tabular}{lllll}
\hline \multirow{2}{*}{$\begin{array}{l}\text { Variáveis independentes e fit do } \\
\text { modelo }\end{array}$} & $\begin{array}{l}\text { Coef. estimado } \\
\text { na AL }\end{array}$ & $\begin{array}{l}\text { Coef. estimado } \\
\text { no Brasil }\end{array}$ & $\begin{array}{l}\text { Coef. estimado } \\
\text { na AL }\end{array}$ & $\begin{array}{l}\text { Coef. estimado } \\
\text { no Brasil }\end{array}$ \\
\cline { 2 - 5 } Renda é suficiente ${ }^{(\mathrm{e})}$ & $1,114 * * *$ & $2,420^{* * *}$ & $1,184 * * *$ & $1,788^{* * *}$ \\
& $(0,050)$ & $(0,632)$ & $(0,097)$ & $(0,484)$ \\
Sente orgulho de seu país ${ }^{(\mathrm{f})}$ & $1,097 * * *$ & 0,375 & $1,440 * * *$ & $2,624 * * *$ \\
N & $(0,094)$ & $(0,661)$ & $(0,114)$ & $(0,726)$ \\
R-quadrado de McFadden & 7.424 & 84 & 6.589 & 139 \\
Log da verossimilhança & 0,074 & 0,237 & 0,094 & 0,246 \\
Critério de Akaike & $-4733,2$ & $-42,6$ & $-1974,6$ & $-58,2$ \\
\% de casos classificados corretamente & 65,6 & 99,193 & 3963,3 & 130,5 \\
\hline
\end{tabular}

Nota. Esta tabela apresenta, para os anos de 2000 e 2009, os coeficientes estimados via regressão logística, tendo como variável dependente a situação do indivíduo que alegou estar Muito Feliz (Muito satisfeito com a vida =1; demais casos $=0$ ). Para cada um dos dois anos foram estimados dois modelos: um considerando todos os países da América Latina e outro apenas os dados do Brasil. Fonte: Elaborado pelos autores com base nos dados coletados.

$* * *$ p-value $<0,01 ; * *$ p-value $<0,05 ; *$ p-value $<0,1$. O Erro-Padrão da estimativa dos coeficientes está entre parênteses. As estimativas foram obtidas a partir de erro-padrão robusto.

(a) Masculino = 1; Feminino = 2; ${ }^{\text {(b) }}$ Expressa em número de anos; ${ }^{(c)}$ Solteiro = 1; Demais status = 0; ${ }^{\text {(d) }}$ Se o indivíduo chegou a frequentar o ensino superior, mesmo que não concluído $=1$; caso alternativo $=0$; ${ }^{\text {(e) }}$ Se a renda é considerada suficiente, mesmo que não poupando, mas sem problemas, assume valor $=1 \mathrm{se}$ sim, e valor $=0$ no caso alternativo; ${ }^{(\mathrm{f})}$ Se sente orgulho de seu país, assume valor $=1 \mathrm{se}$ sim, e valor $=0$ no caso alternativo.

Com a intenção de discutir inicialmente os resultados obtidos para o ano 2000, nota-se que, de forma agregada, em se tratando dos respondentes da América Latina, três variáveis apresentaram efeito significante sobre a probabilidade de reportar-se Muito Feliz: ter frequentado a Universidade $\left(\widehat{\varphi_{5}} \approx\right.$ 0,226; $p<0,01)$, Renda suficiente $\left(\widehat{\varphi_{6}} \approx 1,114 ; p<0,01\right)$ e Orgulho de seu país $\left(\widehat{\varphi_{7}} \approx\right.$ $1,097 ; p<0,01)$. No entanto, no mesmo ano 2000, quando tratados apenas os dados brasileiros $\left(\widehat{\varphi_{7}} \approx 0,375 ; p>0,1\right)$, os resultados não apontaram significância do Orgulho nacional sobre a probabilidade de ser Muito Feliz.

Já quando considerados os resultados obtidos para o ano de 2009, o resultado mais expressivo é o efeito do Orgulho nacional brasileiro sobre a condição de Muito feliz $\left(\widehat{\varphi_{7}} \approx 2,624 ; p<0,01\right)$. Ou melhor, enquanto em 2000 os respondentes brasileiros mostraram-se indiferentes com respeito ao orgulho de ser brasileiro, na oportunidade de reportar-se Muito Feliz, em 2009, não somente passaram a mostrar efeito significante do orgulho nacional sobre o status de Muito feliz, como apresentaram coeficiente superior ao constatado para o agregado da América Latina $\left(\widehat{\varphi_{6}} \approx 1,440 ; p<0,01\right)$.

Ao mesmo tempo, o efeito da Renda sobre o status de Muito feliz foi reduzido, tal como os coeficientes estimados mostram. Finalmente, verifica-se que, no ano de 2009, entre as variáveis independentes, no Brasil, duas mostraram efeito significante sobre a probabilidade de reportar status de Muito feliz, i.e., Renda e Orgulho nacional. A título de ilustração e interpretação dos coeficientes estimados como significantes, a probabilidade de um indivíduo reportar-se como Muito feliz, no ano de 2009 (Tabela 3), na América Latina, poderia ser expressa sob a forma (3), a seguir:

$$
\begin{aligned}
\ln \left(\frac{\pi_{i}}{1-\pi_{i}}\right)= & 1,019-0,013 . \text { Idade }_{i}+0,744 . \text { Universidade }_{i}+1,184 . \text { Renda }_{i} \\
& +1,440 . \text { Orgulho }_{i}
\end{aligned}
$$

E, portanto, a probabilidade $\pi_{i}$, de um indivíduo com idade ao redor de 40 anos, que não chegou a frequentar a Universidade (dummy $=0)$, que julga sua renda familiar insuficiente para atender suas 
necessidades $($ dummy $=0$ ) e que não sente orgulho de seu país (pontuação $=1$ ), sentir-se Muito Feliz, poderia ser calculada conforme a expressão (4) a seguir:

$$
\pi=\frac{1}{1+e^{-\pi}} \therefore \frac{1}{1+2,178^{-(1,019-0,013.35+0,744.0+1,184.0+1,440.0)}} \therefore \pi \cong 59,59 \%
$$

Contudo, se esse mesmo sujeito sentisse orgulho de seu país, a probabilidade de reportar-se Muito Feliz estaria próxima de $81,9 \%$. Ou seja, sofreria um incremento ao redor de 20 pontos percentuais. Este resultado aponta para a associação entre a satisfação com a vida e o crescimento econômico dos países da América Latina, bem com o sentimento de orgulho da nacionalidade, por parte dos indivíduos, tal como discutem Frey e Stutzer (2002), Oswald (1997), assim como de Corbi e Menezes-Filho (2006).

Destaque-se que, contrariamente ao resultado encontrado para o agregado de países da América Latina no ano 2000 (quando o orgulho nacional mostrou-se associado ao status de Muito Feliz), o conjunto de dados coletados no Brasil não indica associação significante entre orgulho de seu país e o status de Muito Feliz, novamente: considerando-se o ano 2000.

A Tabela 4 apresenta os parâmetros estimados para o modelo (2), que contempla apenas o ano de 2009, por motivos de indisponibilidade de dados para o ano 2000. De maneira diferente do modelo (1), o modelo (2) considera aspectos distintos de satisfação com as condições de vida que exercem o papel de Variáveis de Controle $(V C)$. Dito de outra forma, intencionando verificar se os parâmetros das variáveis independentes mantêm efeito marginal similar sobre a probabilidade de o indivíduo reportar estar Muito Feliz, foram empregadas seis variáveis de controle, i.e., satisfação com: (a) Hospitais públicos, (b) Educação pública, (c) Atuação da polícia, (d) Sistema jurídico, (e) Áreas pública e (f) Transporte.

Tabela 4

Modelos Logit para a Probabilidade de o Indivíduo Alegar Estar Satisfeito com a sua Vida (Apenas 2009)

\begin{tabular}{|c|c|c|c|c|}
\hline \multirow[b]{2}{*}{$\begin{array}{l}\text { Variáveis independentes e fit do } \\
\text { modelo }\end{array}$} & \multicolumn{2}{|c|}{ Indivíduo Muito Feliz } & \multicolumn{2}{|l|}{ Indivíduo Feliz } \\
\hline & $\begin{array}{l}\text { Coef. estimado } \\
\text { na AL }\end{array}$ & $\begin{array}{l}\text { Coef. estimado } \\
\text { no Brasil }\end{array}$ & $\begin{array}{l}\text { Coef. estimado } \\
\text { na AL }\end{array}$ & $\begin{array}{l}\text { Coef. estimado } \\
\text { no Brasil }\end{array}$ \\
\hline \multirow[t]{2}{*}{ Constante } & $2,144 * * *$ & $-2,135$ & $0,560 * * *$ & $0,952^{*}$ \\
\hline & $(0,269)$ & $(1,704)$ & 0,109 & $(0,541)$ \\
\hline \multirow[t]{2}{*}{$\operatorname{Sexo}^{(\mathrm{a})}$} & $-0,071$ & $-0,427$ & $-0,010$ & $-0,136$ \\
\hline & $(0,086)$ & $(0,516)$ & $(0,033)$ & $(0,148)$ \\
\hline \multirow[t]{2}{*}{ Idade $^{(b)}$} & $-0,013 * * *$ & 0,001 & $-0,004 * * *$ & 0,001 \\
\hline & $(0,003)$ & $(0,014)$ & $(0,001)$ & $(0,005)$ \\
\hline \multirow[t]{2}{*}{ Solteiro $^{(\mathrm{c})}$} & 0,089 & $-0,094$ & $0,069 *$ & $-0,194$ \\
\hline & $(0,103)$ & $(0,561)$ & $(0,039)$ & $(0,180)$ \\
\hline \multirow[t]{2}{*}{ Frequentou Universidade $\mathrm{d}^{(\mathrm{d})}$} & $0,817 * * *$ & 2,604 & $0,467 * * *$ & $0,682 * * *$ \\
\hline & $(0,167)$ & $(1,629)$ & $(0,056)$ & $(0,260)$ \\
\hline \multirow[t]{2}{*}{ Renda é suficiente ${ }^{(\mathrm{e})}$} & $1,149 * * *$ & $1,784 * * *$ & $0,862 * * *$ & $0,822 * * *$ \\
\hline & $(0,098)$ & $(0,536)$ & $(0,034)$ & $(0,149)$ \\
\hline \multirow[t]{2}{*}{ Sente orgulho de seu país ${ }^{(f)}$} & $1,376 * * *$ & $3,264 * * *$ & $0,811 * * *$ & $1,083^{* * *}$ \\
\hline & $(0,115)$ & $(0,778)$ & $(0,051)$ & $(0,216)$ \\
\hline
\end{tabular}


Tabela 4 (continuação)

\begin{tabular}{lllll}
\hline \multirow{2}{*}{$\begin{array}{l}\text { Variáveis independentes e fit do } \\
\text { modelo }\end{array}$} & $\begin{array}{l}\text { Indivíduo Muito Feliz } \\
\text { na AL. estimado }\end{array}$ & $\begin{array}{l}\text { Coef. estimado } \\
\text { no Brasil }\end{array}$ & $\begin{array}{l}\text { Coef. estimado } \\
\text { na AL }\end{array}$ & $\begin{array}{l}\text { Coef. estimado } \\
\text { no Brasil }\end{array}$ \\
\cline { 2 - 5 } Satisfação com & 0,036 & $-0,112$ & $-0,036$ & $-0,212^{*}$ \\
Hospitais públicos & $(0,062)$ & $(0,371)$ & $(0,022)$ & $(0,115)$ \\
Educação pública & $-0,243^{* * *}$ & $-0,408$ & $-0,061^{* * *}$ & $-0,004$ \\
& $(0,064)$ & $(0,354)$ & $(0,023)$ & $(0,112)$ \\
Atuação policial & 0,001 & $-0,284$ & $-0,009$ & $-0,268^{* *}$ \\
& $(0,061)$ & $(0,387)$ & $(0,023)$ & $(0,120)$ \\
Sistema jurídico & $-0,095$ & 0,386 & $-0,049^{* * *}$ & 0,121 \\
& $(0,058)$ & $(0,245)$ & $(0,021)$ & $(0,089)$ \\
Áreas públicas & $-0,085^{* * *}$ & 0,210 & $-0,045^{* * * *}$ & 0,032 \\
& $(0,021)$ & $(0,176)$ & $(0,010)$ & $(0,048)$ \\
Transporte público & $-0,027$ & 0,208 & $-0,036^{* * *}$ & 0,062 \\
& $(0,030)$ & $(0,236)$ & $(0,012)$ & $(0,051)$ \\
\hline N & 6589 & 139 & 19933 & 1193 \\
R-quadrado de McFadden & 0,110 & 0,291 & 0,053 & 0,075 \\
Log da verossimilhança & $-1940,6$ & $-54,78$ & $-11304,8$ & $-580,5$ \\
Critério de Akaike & 3907,2 & 135,56 & 22635,7 & 1187,02 \\
\% de classificação correta & 89,9 & 82,0 & 71,8 & 78,2 \\
\hline
\end{tabular}

Nota. Esta tabela apresenta os coeficientes estimados para a regressão logística, tendo como variável dependente a situação do indivíduo que alegou estar Muito feliz. Esta tabela reporta os resultados obtidos para o de 2009 (não existiam dados disponíveis para o ano 2000 relativos à satisfação com diferentes serviços públicos). Foi estimado um modelo considerando todos os países da América Latina e outro modelo com dados apenas do Brasil. Fonte: Elaborado pelos autores com base nos dados coletados. $* * *$ p-value $<0,01 ; * *$ p-value $<0,05 ; *$-value $<0,1$. O Erro Padrão da estimativa dos coeficientes está entre parênteses. As estimativas foram obtidas a partir de erro-padrão robusto.

(a) Masculino = 1; Feminino = 2; ${ }^{\text {(b) }}$ Expressa em número de anos; ${ }^{\text {(c) }}$ Solteiro $=1$; Demais status $=0$; ${ }^{\text {(d) }}$ Se o indivíduo chegou a frequentar o ensino superior, mesmo que não concluído $=1$; caso alternativo $=0$; ${ }^{(e)}$ Se a renda é considerada suficiente, mesmo que não poupando, mas sem problemas, assume valor $=1 \mathrm{se}$ sim, e valor $=0$ no caso alternativo; $\mathrm{e}^{(\mathrm{f})}$ Se sente orgulho de seu país, assume valor $=1$ se sim, e valor $=0$ no caso alternativo.

Então, ao considerar a satisfação com serviços públicos, o orgulho do país passou a exercer efeito marginal significante sobre a probabilidade de ser classificado como Muito Feliz ou Feliz, sugerindo que o Orgulho nacional parece ser uma variável relevante para a satisfação com a vida dos indivíduos. E este resultado foi persistente entre o agregado de países da América Latina $\left(\widehat{\varphi_{7}} \approx 1,376 ; p<0,1\right)$ e o Brasil $\left(\widehat{\varphi_{7}} \approx 3,264 ; p<0,1\right)$, quando considerados os indivíduos Muito Felizes e os outros que reportaram se considerar Felizes, com diferentes resultados para Brasil $\left(\widehat{\varphi_{7}} \approx 1,083 ; p<0,1\right) \mathrm{e}$ América Latina $\left(\widehat{\varphi_{7}} \approx 0,811 ; p<0,1\right)$.

Conforme o sugerido pelos coeficientes apresentados na Tabela 4, onde estão considerados os povos da América Latina, no ano de 2009, verifica-se que Educação pública e Áreas públicas exercem efeito significante sobre a satisfação com a vida, tanto entre as pessoas que se apresentaram como Muito Feliz, como entre aquelas que se apresentaram como Feliz. Isto é, a satisfação com Educação pública, entre os países latino-americanos, mostrou-se associada à declaração de Muito Feliz $\left(\widehat{\varphi_{9}} \approx\right.$ $-0,243 ; p<0,01)$ e também no conjunto de pessoas que se disseram Felizes $\left(\widehat{\varphi_{9}} \approx-0,061 ; p<\right.$ $0,01)$. 
De maneira similar, entre os países latino-americanos, o impacto da satisfação com Áreas públicas apresentou-se associada ao status de Muito Feliz $\left(\widehat{\varphi_{12}} \approx-0,085 ; p<0,01\right)$ ou feliz $\left(\widehat{\varphi_{12}} \approx-0,045 ; p<0,01\right)$. De maneira alternativa, nenhum desses resultados foi constatado no conjunto de respondentes brasileiros. Assim, entre as variáveis de controle consideradas, nota-se que a satisfação com a Atuação policial foi a única a mostrar coeficiente significante $\left(\widehat{\varphi_{10}} \approx-0,268 ; p<\right.$ $0,05)$. Os resultados encontrados neste trabalho podem ser semelhantes a estudos anteriores, como os de Mendes-da Silva et al. (2013), Lichtenstein e Slovic (2006), Loewenstein, Read e Baumeister (2003) e Burr et al. (2011), em que abordam que direcionadores de felicidade podem estar associados a: perspectivas de trabalho, oportunidades de crescimento intelectual, situação financeira e segurança pessoal.

\section{Considerações Finais}

Argumentos encontrados na literatura afirmam existir associações significativas entre felicidade (aqui entendido como sinônimo de satisfação com a vida), saúde, renda, orgulho nacional, segurança, entre outros. Nesse sentido, alguns países têm considerado, formalmente, a felicidade na proposição de suas políticas públicas; além disso, empresas e organismos internacionais têm dedicado esforços no melhor entendimento dos determinantes da felicidade. Contudo, especialmente nas economias emergentes, pouco ainda se conhece acerca desse tema, ao mesmo tempo que sua relevância apresentase evidente.

O objetivo desta pesquisa foi verificar a existência de associações entre a Satisfação com a vida (felicidade) e Orgulho nacional, bem como a satisfação com determinados aspectos de vida, uma vez que o estabelecimento de políticas públicas associadas ao tratamento da felicidade aponta para a necessidade de melhor entendimento da subjetividade decorrente do julgamento das pessoas. Para tanto, foram empregados dados coletados pelo Latinobarómetro, no início e no fim da década 2000-2009.

A partir de modelos de resposta binária, os principais resultados encontrados, considerando-se os dados do ano 2000, neste estudo, sugerem que a renda exerce efeito sobre a probabilidade do indivíduo se declarar Muito Feliz, tanto no Brasil como na América Latina. No entanto, na América Latina mais dois fatores foram apontados: ter frequentado a Universidade e Orgulho do país. No Brasil, em 2009, nota-se que o orgulho de ser brasileiro passa a exercer efeito sobre a probabilidade do indivíduo se declarar Muito Feliz, e que, comparado à América Latina, apresenta-se com coeficiente mais elevado $\left(\widehat{\varphi_{7}} \approx 1,440 ; p<0,01\right)$. Este resultado é consistente com os achados de Morrison et al. (2011) e Reeskens e Wright (2011), que relatam a associação positiva entre Orgulho nacional e Satisfação com a vida.

Ao considerar apenas os dados relativos ao ano 2009, considerando o agregado de respostas da América Latina, verificou-se que Educação pública e Áreas públicas exercem efeito persistente sobre a satisfação com a vida. Isto é, a satisfação com Educação pública, entre os países latino-americanos, mostrou-se associada à declaração de Muito Feliz. Nenhum desses resultados foi constatado no conjunto de respondentes brasileiros. Entre as variáveis de controle consideradas, apenas a satisfação com a Atuação policial apresentou coeficiente significante. Ou seja, quanto mais satisfeito com a Atuação da polícia, maior a probabilidade de declarar-se Mais feliz. Esse resultado apoia achados do trabalho de Mendes-da-Silva et al. (2013), segundo o qual a segurança pessoal apresenta-se como aspecto de vida mais relevante para a satisfação com a vida em uma grande metrópole. Em adição, a segurança pública, ainda segundo Mendes-da-Silva et al. (2013), pode contribuir para a ocorrência de vieses de julgamento acerca da propensão a movimentos migratórios. Em outras palavras, a percepção de segurança pessoal pode induzir erros de julgamento acerca da satisfação com a vida em determinadas cidades.

Tendo em vista que os determinantes da felicidade constituem tema carente de mais investigação, este trabalho se apresenta relevante, pois contribui para o melhor entendimento dos determinantes da felicidade, envolvidos nas questões de ordem pública (educação, segurança, mobilidade, entre outros), 
bem como aspectos sociais e demográficos. Um efeito consequente desse argumento é o interesse de alguns países, inclusive o Brasil, em considerar variáveis de felicidade no desempenho da gestão de políticas públicas, além de empresas e organismos internacionais.

O presente estudo possui limitações merecedoras de registro. A primeira delas é a impossibilidade de realizar a análise de dados em painel, uma vez que os dados não oferecem esse recurso. Em segundo lugar, tendo em vista o caráter complexo do tema estudado, existe a possibilidade de viés de variáveis omitidas nos modelos empíricos adotados, o que poderia ser atenuado acaso fosse possível e viável o uso de técnicas de regressão de dados em painel. A terceira limitação que se apresenta relevante é que são empregados dados relativos a dois momentos do tempo, o que pode carregar especificidades do momento político econômico de cada país considerado no survey que possibilitou este estudo. A quarta limitação que se entende deva ser registrada é que não foram explorados, por questões de escopo da pesquisa, os determinantes do Orgulho nacional.

Os resultados obtidos nesta pesquisa juntam-se às limitações do estudo, bem como à relevância do tema aqui estudado, e apontam para uma agenda de pesquisa. Nesse sentido, sugere-se, basicamente, duas linhas de trabalho: (a) replicar o presente estudo, incluindo novas variáveis e analisando outros períodos, buscando verificar a persistência dos resultados aqui obtidos e (b) analisar os determinantes do Orgulho nacional, abordando temas que são, ao mesmo tempo, de elevado interesse na comunidade e pertencentes à cultura brasileira. Ou seja, sugere-se conduzir estudos no sentido de analisar a influência de eventos e desempenho nos esportes e o orgulho de ser brasileiro (Kavetsos, 2012).

\section{Agradecimentos}

O autor Wesley Mendes-da-Silva agradece o apoio financeiro prestado pelo Instituto Mongeral Aegon de Longevidade, ao projeto de desenvolvimento conduzido no âmbito da Escola de Administração de Empresas de São Paulo da Fundação Getulio Vargas (FGV/EAESP), o qual viabilizou parte dos esforços de pesquisa relativos ao presente artigo.

\section{Referências}

Ahuvia, A. C., \& Friedman, D. C. (1998). Income, consumption, and subjective well-being: toward a composite macromarketing model. Journal of Macromarketing, 18(2), 153-168. doi: $10.1177 / 027614679801800207$

Aldrich, J. H., \& Nelson, F. D. (1984). Linear probability, logit, and probit models. Beverly Hills, CA: Sage.

Baer, W. (2014). The Brazilian economy: growth \& development (7th ed.). Boulder: Lynne Rienner Publishers.

Boyce, C. J., Brown, G. D. A., \& Moore, S. C. (2010). Money and happiness: rank of income, not income, affects life satisfaction. Psychological Science, 21(4), 471-475. doi: $10.1177 / 0956797610362671$

Brickman, P., \& Campbell, D. (1971). Hedonic relativism and planning the good society. In M. H. Apley (Ed.), Adaptation-level theory: a symposium (pp. 287-302). New York: Academic Press.

Burr, A., Santo, J. B., \& Pushkar, D. (2011). Affective well-being in retirement: the influence of values, money, and health across three years. Journal of Happiness Studies, 12(1), 17-40. doi: $10.1007 / \mathrm{s} 10902-009-9173-2$ 
Cano, W. (2009). América Latina: notas sobre a crise atual. Economia e Sociedade, 18(3), 603-621. doi: 10.1590/S0104-06182009000300008

Corbi, R. B., \& Menezes-Filho, N. A. M. (2006). Os determinantes empíricos da felicidade no Brasil. Revista de Economia Política, 26(4), 518-536. doi: 10.1590/S0101-31572006000400003

DeNeve, K. M., \& Cooper, H. (1998). The happiness personality: a meta-analysis of 137 personality traits and subjective well-being. Psychological Bulletin, 124(2), 197-229.

Denham, B. E. (2010). Correlates of pride in the performance success of United States athletes competing on an international stage. International Review for the Sociology of Sport, 45(4), 457473. doi: $10.1177 / 1012690210373540$

Diener, E., \& Chan, M. Y. (2011). Happy people live longer: subjective well-being contributes to health and longevity. Applied Psychology: Health and Well-Being, 3(1), 1-43. doi: 10.1111/j.17580854.2010.01045.x

Diener, E., \& Seligman, M. E. P. (2004). Beyond money: toward an economy of well-being. Psychological Science in the Public Interest, 5(1), 1-31.

Dolan, P., Peasgood, T., \& White, M. (2008). Do we really know what makes us happy: a review of the economic literature on the factors associated with subjective well-being. Journal of Economic Psychology, 29(1), 94-122. doi: 10.1016/j.joep.2007.09.001

Duesenberry, J. (1949). Income, saving and the theory of consumer behavior. Cambridge, MA: Harvard University Press.

Easterlin, R. (2001). Income and happiness: towards a unified theory. The Economic Journal, 111(473), 465-484. doi: 10.1111/1468-0297.00646

Edgerton, R. B. (1992). Sick societies: challenging the mynth of primitive harmony. New York: Free Press.

Evans, M. D. R., \& Kelley, J. (2002). National pride in the developed world: survey data from 24 nations. International Journal of Public Opinion Research, 14(3). doi: 10.1093/ijpor/14.3.303

Ferrer-i-Carbonell, A., \& Frijters, P. (2004). How important is methodology for the estimates of the determinants of happiness? The Economic Journal, 114(497), 641-659. doi: 10.1111/j.14680297.2004.00235.x

Frey, B. S., \& Stutzer, A. (2002). What can economists learn from happiness research? Journal of Economic Literature, 40(2), 402-435.

Graham, C. (2008). Happiness and health: lessons, and questions, for public policy. Health affairs, 27(1), 72-87. doi: 10.1377/hlthaff.27.1.72.

Ha, S. E., \& Jang, S. J. (2015). National identity, national pride, and happiness: the case of Korea. Social Indicators Research, 121(2), 471-482. doi: 10.1007/s11205-014-0641-7

Hallmann, K., Breuer, C., \& Kühnreich, B. (2013). Happiness, pride and elite sporting success: what population segments gain most from national athletic achievements? Sport Management Review, 16(2), 226-235. doi: 10.1016/j.smr.2012.07.001

Helliwell, J., Layard, R., \& Sachs, J. (2013). World happiness report 2013. Retrieved from http://unsdsn.org/wp-content/uploads/2014/02/WorldHappinessReport2013_online.pdf 
Inglehart, R. (1996). The diminishing utility of economic growth: from maximizing security toward maximizing subjective well-being. Critical Review: A Journal of Politics and Society, 10(4), 509531. doi: $10.1080 / 08913819608443436$

Jeon, Y., Black, A., Govett, J., Yen, L., \& McRae, I. (2012). Private health insurance and quality of life: perspectives of older Australians with multiple chronic conditions. Australian Journal of Primary Health, 18(3), 212-219. doi: 10.1071/PY11059

Kahneman, D. (1999). Objective happiness. In D. Kahneman, E. Diener, \& N. Schwarz (Eds.), Wellbeing: foundations of hedonic psychology (pp. 3-25). New York: Russell Sage Foundation Press.

Kahneman, D., Krueger, A. B., Schkade, D., Schwarz, N., \& Stone, A. A. (2006). Would you be happier if you were richer? A focusing illusion. Science, 312(5782), 1908-1910. doi: $10.1126 /$ science. 1129688

Kavetsos, G. (2012). National pride: war minus the shooting. Soc Indic Res, 106(1), 173-185, doi: 10.1007/s11205-011-9801-1

Latinobarómetro Corporation. (2000). Latinobarómetro Survey. Recuperado de http://www.latinobarometro.org/latContents.jsp

Latinobarómetro Corporation. (2009). Latinobarómetro Survey. Recuperado de http://www.latinobarometro.org/latContents.jsp

Lichtenstein, S., \& Slovic, P. (2006). The construction of preference. Cambridge: Cambridge University Press.

Loewenstein, G., Read, D., \& Baumeister, R. F. (2003). Time and decision: economic and psychological perspectives on intertemporal choice. New York: Russel Sage Foundation.

London Datastore. (n.d.). London ward well-being scores. Retrieved from http://data.london.gov.uk/dataset/london-ward-well-being-scores

Luz, T. C. B., César, C. C., Lima-Costa, M. F., \& Proietti, F. A. (2011). Satisfaction with the neighborhood environment and health in older elderly: cross-sectional evidence from the Bambuí cohort study of aging. Cadernos de Saúde Pública, 27(3), S390-S398. doi: 10.1590/S0102311X2011001500009

Lykken, D., \& Tellegen, A. (1996). Happiness is a stochastic phenomenon. Psychological Science, 7(3), 186-189. doi: 10.1111/j.1467-9280.1996.tb00355.x

Menard, S. (2002). Applied logistic regression analysis. Thousand Okas, CA: Sage Publications.

Mendes-da-Silva, W., Onusic, L. M., Norvilitis, J. M., \& Moura, G. L. (2013). Focusing illusion in satisfaction with life among college students living in Brazil. Revista de Administração de Empresas, 53(5), 430-441. doi: 10.1590/S0034-75902013000500002

Moller, V., Dickow, H., \& Harris, M. (1999). South Africa's "rainbow people", national pride and happiness. Social Indicators Research, 47(3), 245-280. doi: 10.1023/A:1006945108139

Morrison, M., Tay, L., \& Diener, E. (2011). Subjective well-being and national satisfaction: findings from a worldwide survey. Psychological Science, 22(2), 166-171. doi: $10.1177 / 0956797610396224$

Ng, Y.-K. (1997). A case for happiness, cardinalism, and interpersonal comparability. Economic Journal, 107(445), 1848-1858. doi: 10.1111/j.1468-0297.1997.tb00087.x 
OECD Better Life Index. (2014). OECD regional well-being: a user's guide. Retrieved from http://www.oecdregionalwellbeing.org/assets/downloads/Regional-Well-Being-User-Guide.pdf

Oswald, A. J. (1997). Happiness and economic performance. Economic Journal, 107(445), 1815-1831. doi: 10.1111/j.1468-0297.1997.tb00085.x

Oswald, A. J., Proto, E., \& Sgroi, D. (2014). Happiness and productivity. Retrieved from http://www2.warwick.ac.uk/fac/soc/economics/staff/eproto/workingpapers/happinessproductivit y.pdf

Platt, S., Micciolo, R., \& Tansella, M. (1992). Suicide and unemployment in Italy: description, analysis and interpretation of recent trends. Social Science \& Medicine, 34(11), 1191-1201. doi: 10.1016/0277-9536(92)90312-E

Proposta de Emenda à Constituição $n^{\circ} 19$ de 2010. (2010). Altera o artigo $6^{\circ}$ da Constituição Federal para incluir o direito à busca da Felicidade por cada indivíduo e pela sociedade, mediante a dotação pelo Estado e pela própria sociedade das adequadas condições de exercício desse direito. Recuperado em 19 de outubro, 2015, de http://www25.senado.leg.br/web/atividade/materias//materia/97622

Reeskens, T., \& Wright, M. (2011). Subjective well-being and national satisfaction: taking seriously the "proud of what?" Question. Psychological Science, 22(11), 1460-1462. doi: $10.1177 / 0956797611419673$

Sagiv, L., \& Schwartz, S. H. (2000). Value priorities and subjective well-being: direct relations and congruity effects. European Journal of Social Psychology, 30(2), 177-198. doi: 10.1002/(SICI)1099-0992(200003/04)30:2<177::AID-EJSP982>3.0.CO;2-Z

Schildkraut, D. J. (2014). Boundaries of American identity: evolving understandings of "Us". Annual Review of Political Science, 17(1), 441-460. doi: 10.1146/annurev-polisci-080812-144642

Schkade, D. A., \& Kahneman, D. (1998). Does living in California make people happy? A focusing illusion in judgments of life satisfaction. Psychological Science, 9(5), 340-346. doi: $10.1111 / 1467-9280.00066$

Schwartz, S. H., Sagiv, L., \& Boehnke, K. (2000).Worries and values. Journal of Personality, 68(2), 309-346. doi: 10.1111/1467-6494.00099

Scorsolini-Comin, F., \& Santos, M. A. (2010). O estudo científico da felicidade e a promoção da saúde: revisão integrativa da literatura. Revista Latino Americana de Enfermagem, 18(3), 472-479. doi: 10.1590/S0104-11692010000300025

Serrano, F., \& Summa, R. (2011, agosto). Política macroeconômica, crescimento e distribuição de renda na economia brasileira dos anos 2000. Anais do Encontro Internacional da Associação Keynesiana Brasileira, Rio de Janeiro, RJ, Brasil, 4.

Tajfel, H. (1981). Human groups and social categories. Cambridge: Cambridge University Press.

Veenhoven, R. (2008). Healthy happiness: effect of happiness on physical health and the consequences for preventive health care. Journal Happiness Studies, 9(3), 449-469. doi: 10.1007/s10902-0069042-1 


\section{Dados dos Autores}

Luciana Massaro Onusic

Rua Angélica, 100, Jardim das Flores, 06110-295, Osasco, SP, Brasil. E-mail: lucianaonusic@ gmail.com

Wesley Mendes-da-Silva

Rua Itapeva, 474, 01332-000, São Paulo, SP, Brasil. E-mail: mr.mendesdasilva@ gmail.com 